\title{
Some Remarks on Charge Densities in Bent Bond Molecules*
}

\author{
OLLE MARTENSSON and GUNNAR SPERBER
}

\author{
Quantum Chemistry Group, Uppsala University, Box 518, S.751 20 Uppsala 1
}

\begin{abstract}
The electron densities obtained from extended Hückel calculations including $s$ and $p$ orbitals are studied for the strained molecules cyclopropane and $\mathrm{P}_{4}$. The difference electron density of cyclopropane obtained is in good agreement with experimental results from X-ray investigations of molecules containing this ring system, which indicate maxima outside the $\mathrm{C}-\mathrm{C}$ lines.

The same type of difference density was obtained for the $P-P$ bond in $\mathbf{P}_{\mathbf{4}}$. The total valence electron density showed distinct maxima outside the corners of the tetrahedron, indicating the occurrence of lone pairs in the $\mathbf{P}$ atoms.

The chemically interesting symbol problem "straight lines for straight bonds; bent lines for bent bonds" and the concept of bond length are also commented on.
\end{abstract}

\begin{abstract}
$\mathrm{T}$ he use of a line joining two atoms as a symbol for a chemical bond is more than a hundred years old. It was introduced by Couper in $1858^{1}$ and is still used. By this symbol it became possible to write structure formulas, describing how the atoms were joined together and explain structure isomerism. However, the spatial arrangement of the atoms in a molecule was considered later and culminated in the discovery by van't Hoff and by le $\mathrm{Bel}^{2}$ of the tetrahedral orientation of the four valences of carbon. The introduction of other types of bonds, viz. the double and the triple bond, also made it possible to explain cis-trans isomerism (and so represent these bonds by simple symbols, the double line and the triple line). Thus, spatial properties could be ascribed to the straight line serving as a symbol for a bond and it became a representation for a directed valence. The discovery of the electron opened new perspectives to the explanation of the chemical bond and in 1916 the octet theory was developed by Lewis and by Kossel. ${ }^{3}$ Lewis' interpretation of the covalent bond as an electron-pair formation, and his replacement of the straight line by two dots, can be said to be the first attempt to utilize the electron density in the formal description of the covalent bond, although the modern quantum theory first made such an interpretation possible. The
\end{abstract}

\footnotetext{
* Sponsored in part by King Gustaf VI Adolf's 80-Years' Fund for Swedish Culture, Knut and Alice Wallenberg's Foundation, and in part by the Swedish Natural Science Research Council.
}

Acta Chem. Scand. 24 (1970) No. 5 
directed valence is conventionally described by means of hybrids built up as linear combinations of rather few atomic orbitals (generally, and here unless otherwise stated, of $s$ and $p$ type). The two hybrids forming a bond are thus directed along the internuclear vector, we get a "straight" bond, and the "track of highest electron density" will coincide with the internuclear vector.

Since conventional hybridization does not permit the description of bond angles less than $90^{\circ}$, bonds in so called strained molecules such as cyclopropane have been difficult to interpret. It has been necessary to introduce so-called "bent bonds", i.e. the bonds are described by hybrids, given a direction different from that of the internuclear vector. The direction of a hybrid is then generally determined by some version of the maximum overlap method. The idea of bent bonds is far from new. Indeed, it was the first attempt to describe the ordinary double bond with its cis-trans (geometrical) isomerism. The bent bond idea has been intensely discussed and the literature about bent bonds and related topics is extensive. Here we will merely refer to the handbooks by Coulson and by Pauling and to some recent papers. ${ }^{4-6}$

\section{THE CONCEPT OF BENT BOND AND BOND LENGTH}

If one tries to give a quantum mechanical interpretation of the classical directed valence and its symbol as they are used in the ordinary structure formulas of the organic chemists, one has to remember that they represent some kind of nearest-neighbor approximation and that an approach beyond this approximation in all probability will be of less interest since the main importance of the problem is on the conceptual side.

In an ordinary "straight" bond one expects the track of maximum charge density (electron density) to lie approximately on the straight line joining the two nuclei. In a bent bond, however, one cannot expect this to be the case. Some results from $\mathrm{X}$-ray investigations of compounds containing a cyclopropane ring confirm that the charge density maxima of the $\mathrm{C}-\mathrm{C}$ bonds are displaced in the expected direction. ${ }^{7,8}$

Therefore in this paper we will examine theoretically the charge densities of the bent bond molecules cyclopropane $\left(\mathrm{C}_{3} \mathrm{H}_{6}\right)$ and tetrahedral phosphorus $\left(\mathrm{P}_{4}\right)$, within some simple approximations of the LCAO type. We will also discuss the possibility of defining "bond lines" for such bonds as tracks of maximum charge density. Such lines might be considered as a possible generalization of the classical "valence line" concept. In other words, straight bonds are to be described by straight lines, and bent bonds by bent lines. The question is, whether such generalized bond lines can be suitably defined by considering charge densities, and in particular whether the resulting "bond lengths" make any sense from chemical points of view.

If we will retain the concept of bond length (which is far from necessary, since it is the internuclear distance which is of prime interest) we have to give it a definition as the length of the curve which symbolizes the bond. This curve can be defined in different ways. The nearest choice is perhaps to select the curve representing the points of highest electron density between the two atomic centers, which in turn perhaps is best determined by means of Fermat's 
principle (the variational principle). If $\mathrm{A}$ and $\mathrm{B}$ are the two atomic centers, and $\varrho$ the electron density function, the curve might then be determined by the variational condition $\delta \int_{\mathrm{A}}{ }^{\mathrm{B}}(1 / \varrho) \mathrm{d} s=0$. It is obvious that this curve, representing the bond, in its shape will depend on the kind and approach of the density function. We can select a density function based on all electrons, on the valence electrons, on the $\sigma$-electrons and on the $\pi$-electrons, depending on what we are interested in. But one has also to decide whether one shall consider a density function as a whole, or subtract the atomic contributions from it. That means that one considers the difference density functions as being of main interest in the bonding. For comparison with $\mathrm{X}$-ray data, an all-electron treatment with subtraction of atomic contributions from the core and valence electrons perhaps would be the best choice.

\section{CYCLOPROPANE}

The bonds of particular interest in this context are the $\mathrm{C}-\mathrm{C}$ bonds. Since the $\mathrm{H}-\mathrm{C}-\mathrm{H}$ angle is about $118^{\circ}$, the carbon atoms are usually supposed to be in a "not quite tetrahedral" state of hybridization, leading to an angle of about $100^{\circ}$ between the hybrids which take part in the $\mathrm{C}-\mathrm{C}$ bonds.

Our first attempt to describe these $\mathrm{C}-\mathrm{C}$ bonds will be a naive "orbital pair approximation". The two real hybrids used for this purpose, $h_{\mathrm{A}}$ and $h_{\mathrm{B}}$, are supposed to be built up from ordinary Slater atomic orbitals, are normalized and have the overlap integral $S=\int h_{\mathrm{A}} h_{\mathrm{B}} \mathrm{d} v$. If we now treat the two electrons which form the bond as isolated from and non-interacting with the rest of the molecule, we may apply the simple MO or VB approximations for a homonuclear, diatomic two electron molecule. The MO theory gives us the following "electron pair charge density"

$$
\varrho_{\mathrm{MO}}=\left[h_{\mathrm{A}}^{2}+h_{\mathrm{B}}^{2}+2 h_{\mathrm{A}} h_{\mathrm{B}}\right] /(1+S)
$$

and the VB theory the slightly different

$$
\varrho_{\mathrm{VB}}=\left[h_{\mathrm{A}}^{2}+h_{\mathrm{B}}^{2}+2 S h_{\mathrm{A}} h_{\mathrm{B}}\right] /\left(1+S^{2}\right)
$$

From either of these we may form a "difference density" by subtracting the density $h_{\mathrm{A}}{ }^{2}+h_{\mathrm{B}}{ }^{2}$ which we get by putting one electron in each hybrid orbital without letting these interact.

From the MO density we thus get the difference density

$$
\Delta_{\mathrm{MO}}=\left[-S\left(h_{\mathrm{A}}^{2}+h_{\mathrm{B}}^{2}\right)+2 h_{\mathrm{A}} h_{\mathrm{B}}\right] /(1+S)
$$

and $\Delta_{\mathrm{VB}}$ is simply $\Delta_{\mathrm{MO}} \cdot S(1+S) /\left(1+S^{2}\right)$.

For simplicity and to accentuate the effect of bond bending, we have here supposed the hybrids to be of the tetrahedral $\left(s p^{3}\right)$ type, and consequently we have let the angles between the hybrid axes and the internuclear vector be $25^{\circ}$. The equilibrium distance between the carbon nuclei is about 2.9 a.u. ( 1 a.u. $=0.529 \AA$ ), and the overlap $S$ is 0.60 . In Figs. $1-3$ we display $\varrho_{\text {мо }}$, $\varrho_{\mathrm{VB}}$, and $\Delta_{\mathrm{MO}}$ in the plane through the hybrid axes as contour diagrams. These contours were found automatically by a computer program, starting from a rectangular net of function values (also calculated by the computer) 


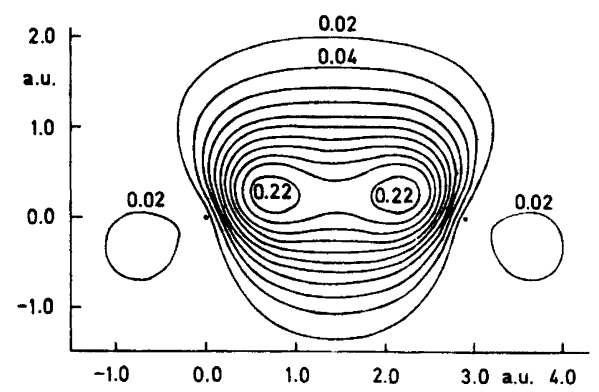

Fig. 1. The charge density $\varrho_{\mathrm{MO}}$ of a bonding electron pair of cyclopropane, in the plane of the carbon nuclei. The nuclei are marked by dots.

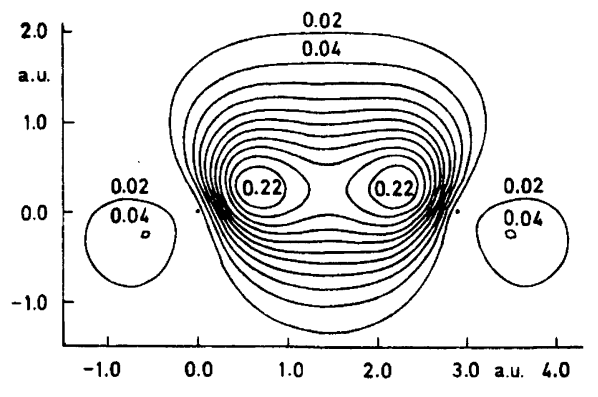

Fig. 2. The charge density $\varrho_{\mathrm{vB}}$ of a bonding electron pair in cyclopropane.

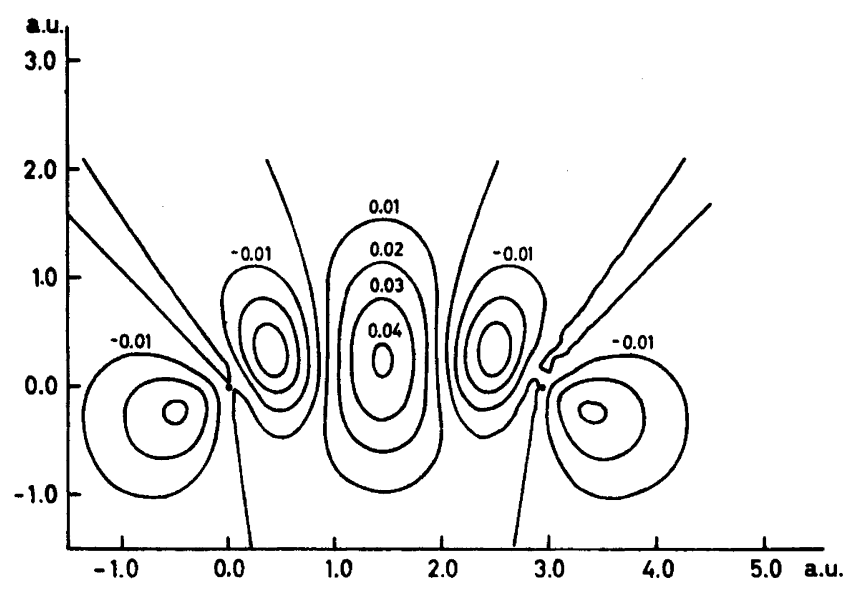

Fig. 3. The difference density $\Delta_{\text {MO }}$ of a bonding electron pair in cyclopropane.

and using interpolation of the second order. The mesh size of the net was 0.15 a.u. for cyclopropane. The slight irregularity of the curves suggest that a smaller mesh size would have been appropriate. $\varrho_{\mathrm{Mo}}$ and $\varrho_{\mathrm{VB}}$ have two maxima in the bonding region, each of them situated at a distance of about $0.4 \AA$ from the nearest atomic center, and the radius vector to it forms an angle of about $20^{\circ}$ with the internuclear line. A simple visualization of the bond by a line of maximum density would give us almost straight lines from the nuclei to the nearest maxima which in turn have to be joined by an almost straight line at a distance of about $0.15 \AA$ from the internuclear axis. The length of this bond would be somewhat more than $1.6 \AA$. (the internuclear distance is $1.53 \AA$ ). The maximum of $\Delta_{\mathrm{MO}}$ is situated very close to this bond line.

Intuitively, we would perhaps expect such a bent bond to be more smoothly curved than its bond line indicates. Also, the maximum of $\Delta_{\text {MO }}$ would be 
expected to lie considerably farther from the bond axis. Although our calculation shows the latter expectation to be founded on a misunderstanding of the nature of the densities involved, experiment happens to bear it out. ${ }^{7,8}$ It is therefore clear (if the experimental results are correct and applicable to cyclopropane itself) that our theoretical model is too crude and must be improved.

A first attempt in this direction is to include the effects of the other bonds in the molecule, simply by adding difference densities arising from the various bonding hybrid pairs. We have done that, again assuming $s p^{3}$ hybridization of the carbon atoms and supposing the $\mathrm{C}-\mathrm{H}$ bonds to be homopolar like the $\mathrm{C}-\mathrm{C}$ bonds. The resulting difference density is shown in Fig. 4. A comparison with Fig. 2. in Ref. 8 quickly convinces us that this refinement has done little to improve the agreement between theory and experiment.

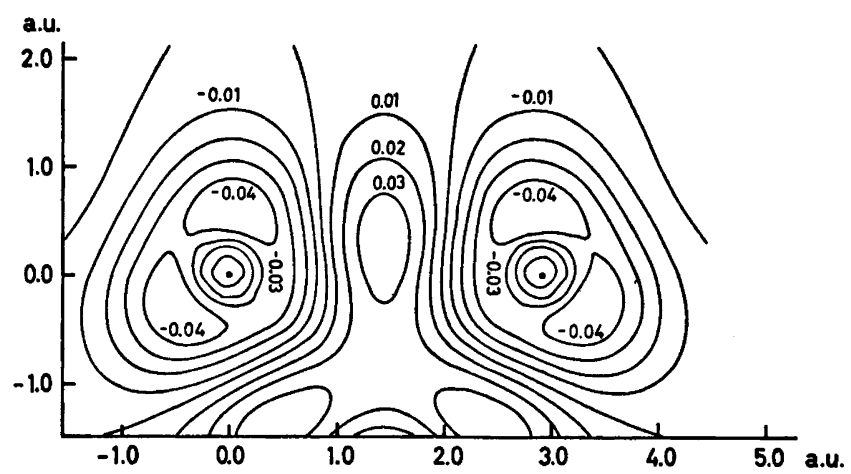

Fig. 4. Difference density for the cyclopropane molecule, in the "sum of bonding electron pairs" approximation.

Several objections may be raised against the procedure described above. In particular, it completely neglects the nonorthogonality integrals for pairs of hybrids that are centered on different atoms but do not interact with each other forming a bond. We therefore chose to do an extended Hückel calculation, using the "charge iterative" modification by Karlsson and Mårtensson," but with a "charge iteration" of second order, which simply means that the expression (13) of Ref. 9 for the Coulomb integrals should be replaced by a quadratic expression in $q$. The coefficients used in this expression are in decreasing order of $q$ :

$\begin{array}{rrrrr}-1.2435 & 21.6975 & -86.3170 & \text { for carbon } 2 s \text { orbitals } \\ -1.6850 & 24.9145 & -84.3780 & \text { " } & 2 p \\ 0.0000 & 12.8500 & -26.4500 & \text { " hydrogen } 1 s "\end{array}$

The constant in the Wolfsberg-Helmholz formula is 1.75. The geometry applied for cyclopropane is: $\mathrm{C}-\mathrm{C}$ distance $1.53 \AA$; $\mathrm{C}-\mathrm{H}$ distance $1.09 \AA$; the $\mathrm{H}-\mathrm{C}-\mathrm{H}$ angle $118.2^{\circ} .10$

The extended Hückel method, which is a simple, semiempirical, delocalized MO-LCAO method, has the property, which is important in this Acta Chem. Scand. 24 (1970) No. 5 
context, of taking all the overlap integrals between valence orbitals into account in an explicit and consistent way. It should be observed that the method makes no explicit use of hybridization at all and that the results therefore contain no "bias" due to assuming any particular hybridization state of the atoms involved. The total density of valence electrons in the plane of the carbon atoms is shown by a level diagram in Fig. 5. As in the

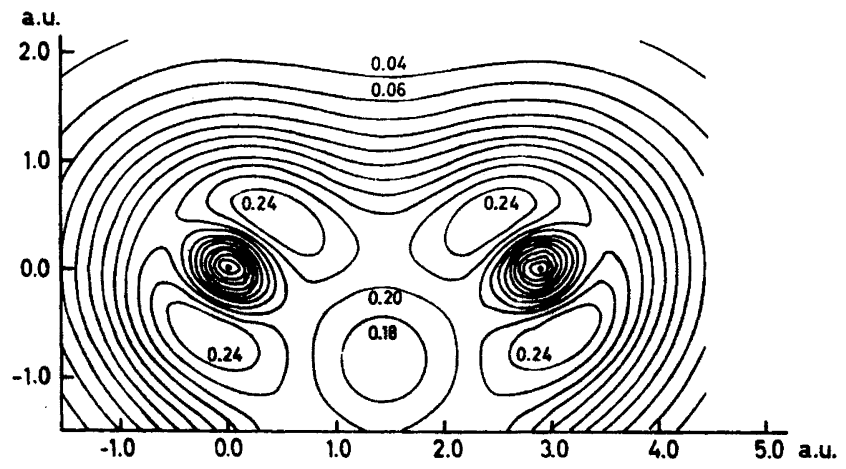

Fig. 5. The valence electron density of the cyclopropane molecule in the plane of the carbon atoms, calculated with the charge iterative extended Hückel method.

case above we find here two maxima, but they are more distant (about $0.35 \AA$ ) from the interatomic line. The radius vector to them from the carbon atoms forms an angle of almost $45^{\circ}$ with the interatomic line. If we draw a bond line according to the maximum density idea, its part between the two maxima will be definitely curved towards the interatomic line. The bond line so drawn will increase the bond length still more and will certainly have a still stranger appearance to the chemist than that of the preceding example.

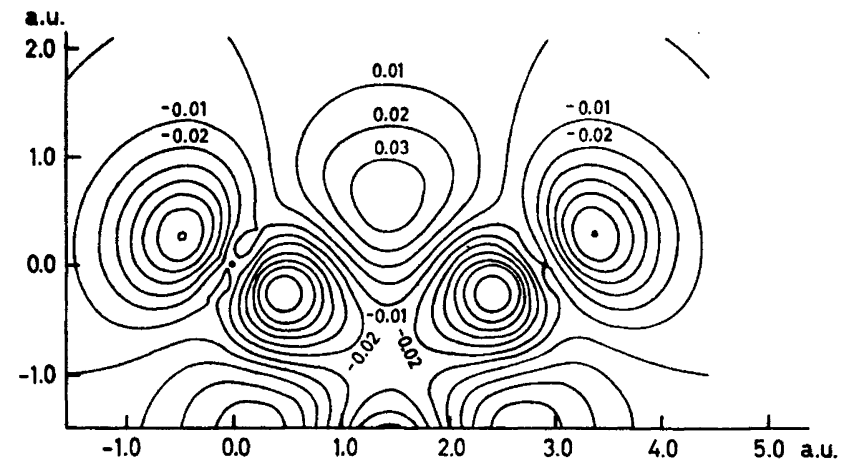

Fig. 6. Difference density of the cyclopropane molecule in the charge iterative extended Hückel method. 
The difference density (Fig. 6) was computed by subtraction of all the atomic Slater orbital densities. We should perhaps note here that the atomic scattering factors for the carbon atoms used to compute the difference density in Ref. 8 were calculated from Hartree-Fock orbitals for carbon.11

Comparing with Fig. 2 in Ref. 8, we see that the qualitative agreement between the theoretical and experimental difference densities is good in this case. The maximum is about $0.3 \AA$ outside the $\mathrm{C}-\mathrm{C}$ bond axis. The minima inside the equilateral triangle (about $0.25 \AA$ from the carbon nuclei), and the slightly negative value at the center, are all qualitatively reproduced. In fact, the agreement is about as good as could be expected considering the following factors:

1. The theoretical density is calculated for a somewhat different molecule than the experimentally investigated one.

2. The experimental density cannot be supposed to be free from errors.

3. Many approximations are inherent in the theoretical treatment, notably the use of a "minimal basis set" of atomic orbitals.

Very recently electron density diagrams for cyclopropane based on $a b$ initio SCF-LCAO-MO calculations have been published by Buenker and Peyerimhoff ${ }^{12}$ and by Kochanski and Lehn. ${ }^{13}$ Their results (see especially Fig. 4 of Ref. 12 and Fig. la of Ref. 13) are not directly comparable with ours, since our valence shell molecular orbitals are not orthogonal to the core orbitals (which are not included in our calculations at all). The general picture of the bond electron density seems to be the same. Unfortunately no difference densities have been reported in the papers mentioned, so a comparison regarding this feature cannot be made.

\section{THE P、 MOLECULE}

Our interest in this molecule arose both from its strained tetrahedral structure and from the fact that Hart, Robin and Kuebler ${ }^{14}$ calculated a valence electron density (based only on the $12 p$ electrons and using unhybridized $3 p$ orbitals) which has some very surprising features. We chose to carry out an extended Hückel calculation including $3 s$ and $3 p$ orbitals and all the 20 valence electrons. (For a homonuclear molecule like $\mathbf{P}_{4}$, the iterative version of the method used for cyclopropane will give the same result as the ordinary, non-iterative method.) The constant in the Wolfsberg-Helmholz formula is 1.75 as in the iterative case. The edge of the $\mathbf{P}_{4}$ tetrahedron is $2.21 \AA . .^{15}$

The results shown are: (i) the valence electron density in a plane containing three nuclei (Fig. 7); (ii) the same density in a plane through two nuclei and the midpoint of the opposite edge (Fig. 8); (iii) a difference density (defined below) in the same plane (Fig. 9).

We see at once that our results have little in common with those of Hart, Robin and Kuebler. A dominant feature of Figs. 7 and 8 is the accumulation of charge outside the corners of the tetrahedron. In addition, there are regions of high density parallel to and slightly outside the edges of the tetrahedron. Both these features agree with a simple intuitive model of the molecule, ac- 


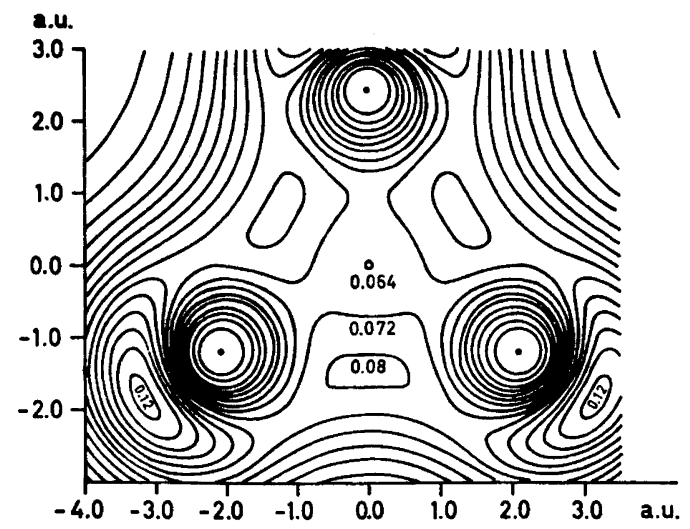

Fig. 7. The valence electron density of the $P_{4}$ molecule in a plane through three nuclei, calculated with the extended Hückel method.

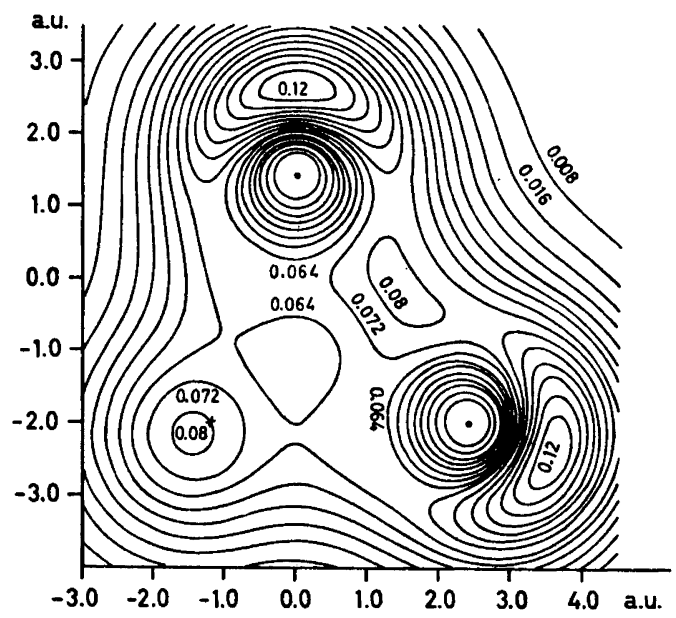

Fig. 8. The valence electron density of $\mathrm{P}_{4}$ in a plane through two nuclei and the midpoint of the opposite edge.

cording to which each atom is in a state of $s p^{3}$ hybridization, two electrons occupying the hybrid directed outward, the other three taking part in bent electron pair bonds outside the edges.

The difference density in Fig. 9 was computed assuming that the unperturbed atoms are already in a state of $s p^{3}$ hybridization with the outward directed hybrid orbital doubly occupied (and thus not in their ground state configuration). We see that the difference density computed in this way behaves more or less analogously to the one for cyclopropane (Fig. 5). 


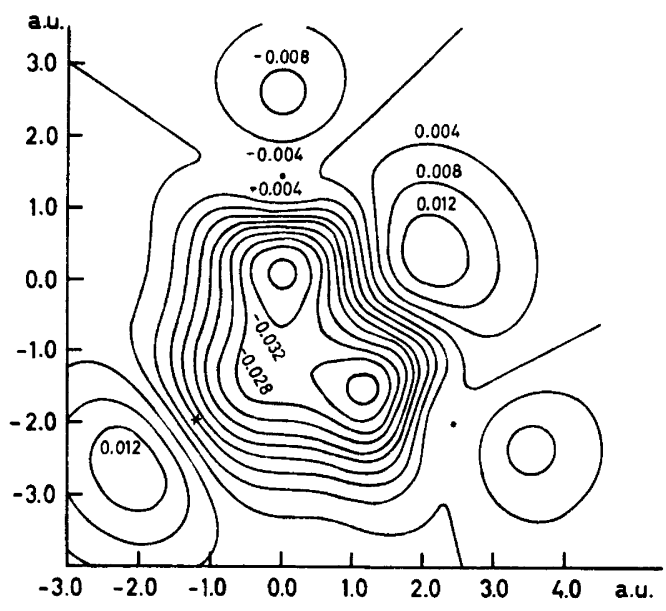

Fig. 9. Difference density of $\mathrm{P}_{4}$ in a plane through two nuclei and the midpoint of the opposite edge, calculated with the extended Hückel method.

\section{CONCLUDING REMARKS}

The results show that in the strained molecules cyclopropane and $\mathrm{P}_{4}$ a considerable amount of the electron density is concentrated outside the interatomic axis of the strained bond. This is of particular interest, since the extended Hückel method does not provide any directed hybrids at all. The same can be said about the lone pair density outside the corners of the $\mathbf{P}_{4}$ tetrahedron. In main the results are in agreement with the intuitive picture one gets from the application of directed hybrids of $s p^{3}$ hybridization. The deviation from the tetrahedral angle is also surprisingly small when hybrids are used in connection with some version of the maximum overlap criterion. There are reasons to believe that more sophisticated calculations of the electron density, at least within the Hartree-Fock scheme, will not change the general picture obtained by the extended Hïckel calculations appreciably.

As to "bond length", it is obvious that it will be difficult to retain this concept in connection with bent bonds, since it demands the definition of a curve symbolizing the bent bond with all its ambiguities. It is also of no practical interest to know this bond length.

Acknowledgements. The authors express their thanks to Drs. G. Karlsson and R. Manne for putting to our disposal their versions of extended Hückel programs, and to members of the Biological Structure Group of the Wallenberg Laboratory for discussions of X-ray electron density diagrams. The calculations and plotting of density diagrams have been carried out on the CD 3600 computer of the Uppsala Data Center. We are very grateful to Prof. P.-O. Löwdin for his support and inspiration. 


\section{REFERENCES}

1. Couper, A. S. Compt. Rend. 46 (1858) 1157; Ann. Chim. Phys. 53 (1858) 469.

2. van't Hoff, J. H. Arch. Neerland. Sci. 9 (1874) 445; le Bel, J. A. Bull. Soc. Chim. France 22 (1874) 337.

3. Lewis, G. N. J. Am. Chem. Soc. 38 (1916) 762; Kossel, W. Ann. Physik. 49 (1916) 229.

4. Pauling, L. The nature of the chemical bond, 3rd Ed., Cornell University Press, Ithaca, N.Y. 1960.

5. Coulson, C. A. Valence, 2nd Ed., Oxford University Press 1961.

6. Coulson, C. A. and Moffitt, W. E. Phil. Mag. 40 (1949) 1; Handler, G. S. and Andersson, J. H. Tetrahedron 2 (1958) 345; Muller, N. and Pritchard, D. F. J. Chem. Phys. 31 (1959) 1471; Murrell, J. N. J. Chem. Phys. 32 (1960) 767; Gilbert, T. L. and Lukos, P. G. J. Chem. Phys. 34 (1961) 2199; Weltner, W., Jr. J. Am. Chem. Soc. 75 (1953) 4224; Coulson, C. A. and Goodwin, T. N. J. Chem. Soc. 1962 2851; 1963 3161; Del Re, G. Theoret. Chim. Acta (Berl.) 1 (1963) 188; Veillard, A. and Del Re, G. Ibid. 2 (1964) 55; Del Re, G., Esposito, U. and Carpentieri, M. Ibid. 6 (1966) 36; Pomerantz, M. and Abrahamsson, E. W. J. Am. Chem. Soc. 88 (1966) 3970; Randić, M. and Maksić, Z. Theoret. Chim. Acta (Berl.) 3 (1965) 59; Klasinc, L., Maksic, Z. and Randic, M. J. Chem. Soc. A 1966 755; Maksić, Z., Klasinc, L. and Randič, M. Theoret. Chim. Acta (Berl.) 4 (1966) 273; Randić, M., Jerkunica, J. M. and Klasinc, L. Ibid. 6 (1966) 240; McWeeny, R. and Del Re, G. Ibid. 10 (1968) 13; Randič, M. and Borčič, S. J. Chem. Soc. A 1967 586; Randic, M. and Stefanović, D. J. Chem. Soc. B 1968 423; Randić, M. and Majerski, Z. Ibid. 1289.

7. Fritchie, C. J., Jr. Acta Cryst. 20 (1966) 27.

8. Hartman, A. and Hirsfeeld, F. L. Acta Cryst. 20 (1966) 80.

9. Hoffman, R. J. Chem. Phys. 39 (1963) 1397; Karlsson, G. and Mårtensson, O. Theoret. Chim. Acta (Berl.) 13 (1969) 195.

10. Sutton, L. E., (Sci. Ed.), Tables of interatomic distances, Spec. publ. 11 (1958) and 18 (1965), London The Chem. Soc. Burlington House, W.L.

11. Berghuis, J., Haanappel, I. J. M., Potters, M., Loopstra, B. O., MacGillavry, C. H. and Veenendaal, A. L. Acta Cryst, 8 (1955) 478.

12. Buenker, R. J. and Peyerimhoff, S. D. J. Phys. Chem. 73 (1969) 1299.

13. Kochanski, E. and Lehn, J. M. Theoret. Chim Acta (Berl.) 14 (1969) 281.

14. Hart, R. R., Robin, M. B. and Kuebler, N. A. J. Chem. Phys. 42 (1965) 3631.

15. Gmelin. Handbuch der anorganischen Chemie, 16 II B. Verlag Chemie Weinheim 1964.

Received December 3, 1969. 